\title{
Fast Learning with Weak Synaptic Plasticity
}

\author{
Pierre Yger, ${ }^{1,2,3} @$ Marcel Stimberg, ${ }^{2,3}$ and Romain Brette ${ }^{2,3}$ \\ ${ }^{1}$ Institut d'Etudes de la Cognition, Ecole Normale Supérieure, 75005 Paris, France, ${ }^{2}$ Sorbonne Université, UPMC Université Paris 06 UMRS968, 75006 Paris, \\ France, and ${ }^{3}$ Institut de la Vision, INSERM U968, CNRS UMR7210, 75012 Paris, France
}

New sensory stimuli can be learned with a single or a few presentations. Similarly, the responses of cortical neurons to a stimulus have been shown to increase reliably after just a few repetitions. Long-term memory is thought to be mediated by synaptic plasticity, but in vitro experiments in cortical cells typically show very small changes in synaptic strength after a pair of presynaptic and postsynaptic spikes. Thus, it is traditionally thought that fast learning requires stronger synaptic changes, possibly because of neuromodulation. Here we show theoretically that weak synaptic plasticity can, in fact, support fast learning, because of the large number of synapses $N$ onto a cortical neuron. In the fluctuation-driven regime characteristic of cortical neurons in vivo, the size of membrane potential fluctuations grows only as $\sqrt{N}$, whereas a single output spike leads to potentiation of a number of synapses proportional to $N$. Therefore, the relative effect of a single spike on synaptic potentiation grows as $\sqrt{N}$. This leverage effect requires precise spike timing. Thus, the large number of synapses onto cortical neurons allows fast learning with very small synaptic changes.

Key words: learning; network; perceptual learning; spike-timing-dependent plasticity

\section{Significance Statement}

Long-term memory is thought to rely on the strengthening of coactive synapses. This physiological mechanism is generally considered to be very gradual, and yet new sensory stimuli can be learned with just a few presentations. Here we show theoretically that this apparent paradox can be solved when there is a tight balance between excitatory and inhibitory input. In this case, small synaptic modifications applied to the many synapses onto a given neuron disrupt that balance and produce a large effect even for modifications induced by a single stimulus. This effect makes fast learning possible with small synaptic changes and reconciles physiological and behavioral observations.

\section{Introduction}

New sensory stimuli can be learned with just a few presentations (Seitz, 2010). For example, children can acquire a new word with a single presentation (Carey and Bartlett, 1978). In vision, fast perceptual learning has also been shown to occur over 10-100 presentations for different tasks such as shape discrimination (Rubin et al., 1997), hyperacuity (Poggio et al., 1992; Fahle et al., 1995), and face identification (Hussain et al., 2012). In audition, $500 \mathrm{~ms}$ tokens of white noise can be discriminated after just a few presentations in an implicit learning task, and the memory remains after several weeks (Agus et al., 2010). In auditory (Fritz et al., 2003) and visual (Yao et al., 2007) cortex, responses of neurons can also change rap-

Received Feb. 13, 2015; revised Aug. 13, 2015; accepted Aug. 19, 2015.

Author contributions: P.Y. and R.B. designed research; P.Y. and R.B. performed research; P.Y., M.S., and R.B. contributed unpublished reagents/analytic tools; P.Y., M.S., and R.B. analyzed data; P.Y., M.S., and R.B. wrote the paper.

This work was supported by the Fondation pour la Recherche Médicale and Agence Nationale de la Recherche (Grant ANR-14-CE13-0003).

The authors declare no competing financial interests.

Correspondence should be addressed to Pierre Yger, Institut de la Vision, INSERM U968, CNRS UMR7210, 75012 Paris, France. E-mail: pierre.yger@inserm.fr.

DOI:10.1523/JNEUROSCI.0607-15.2015

Copyright $\odot 2015$ the authors $\quad 0270-6474 / 15 / 3513351-12 \$ 15.00 / 0$ idly after exposure to sensory stimuli. In particular, when a natural movie is presented a few times, cortical neurons start to fire reliably at specific times, and the specific response persists after other visual stimuli have been presented (Yao et al., 2007).

Long-term memory is thought to be mediated by synaptic plasticity (Whitlock et al., 2006; Nabavi et al., 2014). But in contrast with behavioral and in vivo physiological observations, in vitro experiments on synaptic plasticity in cortical cells reveal very small changes after a pair of presynaptic and postsynaptic spikes: the size of a postsynaptic potential typically increases by around $0.5-5 \%$ when a postsynaptic spike shortly follows a presynaptic spike (Markram et al., 1997; Bi and Poo, 1998; Sjöström et al., 2001; Wang et al., 2005; Caporale and Dan, 2008; for the average over a large number of pairings, see Discussion). In addition, only synapses where a presynaptic spike occurs shortly before the postsynaptic spike are potentiated ( $\simeq 20 \mathrm{~ms}$; Gerstner et al., 1996; Markram et al., 1997; Bi and Poo, 1998), which may concern only a small fraction of them if most inputs are asynchronous during alert states (Renart et al., 2010; Tan et al., 2014). Finally, most cortical neurons fire at a low firing rate (Griffith and Horn, 1966; Koch and Fuster, 1989; Laughlin, 2001; Koulakov et al., 2009), and spontaneous or evoked activities do not normally exceed a 
few tens of hertz. Therefore, a single sensory stimulus lasting 500 ms would not be expected to trigger substantial changes in synaptic strength. Accordingly, in computational models of learning with weak synaptic plasticity, neurons start to respond selectively to a repeated stimulus only after a large number of presentations (Song and Abbott, 2001; Masquelier et al., 2008; Masquelier et al., 2009; Klampfl and Maass, 2013).

Thus, it is traditionally thought that fast learning requires stronger synaptic changes than usually observed in vitro, possibly because of neuromodulation (Fusi et al., 2005). Here we show theoretically that weak synaptic plasticity can, in fact, support fast learning. The mechanism relies on the notion that in vivo, total excitatory input to a cortical neuron is large and matched by inhibition (Softky and Koch, 1993; Destexhe and Paré, 1999; Okun and Lampl, 2008) in the so-called balanced regime. A small relative change in excitation at specific times of the sensory input can then produce a significant difference between the excitatory and the inhibitory drives, i.e., a misbalance resulting in high spiking probability. We show that the leverage effect caused by the excitatory-inhibitory balance is of order $\sqrt{N}$, where $N$ is the number of incoming synapses.

\section{Materials and Methods}

Neuron model. Simulations were performed using current-based, leaky integrate-and-fire neurons with membrane time constant $\tau_{\mathrm{m}}=5 \mathrm{~ms}$ (Destexhe and Paré, 1999) and resting membrane potential $V_{\text {rest }}=-70$ $\mathrm{mV}$. When the membrane potential $V_{\mathrm{m}}$ reaches the spiking threshold $V_{\text {thresh }}=-55 \mathrm{mV}$, a spike is generated, and the membrane potential is clamped to the reset potential $V_{\text {reset }}=-70 \mathrm{mV}$ during a refractory period of duration $\tau_{\text {ref }}=5 \mathrm{~ms}$. Each presynaptic spike produces an exponential decaying current with time constant $\tau_{\mathrm{exc}}=3 \mathrm{~ms}$ for excitation and $\tau_{\mathrm{inh}}=10 \mathrm{~ms}$ for inhibition. The model equations are thus as follows:

$$
\begin{gathered}
\tau_{m} \frac{d V(t)}{d t}=\left(V_{\text {rest }}-V(t)\right)+\mathrm{g}_{\text {exc }}(t)+\mathrm{g}_{\text {inh }}(t) \\
\tau_{\text {syn }} \frac{d g_{\text {syn }}(t)}{d t}=-g_{\text {syn }}(t)
\end{gathered}
$$

where syn $\varepsilon\{$ exc, inh $\}$ and each presynaptic spike produces an instantaneous increase: $g_{\text {syn }} \rightarrow g_{\text {syn }}+\lambda_{\text {syn }} w_{\mathrm{i}}$, where $w_{\mathrm{i}}$ is the synaptic weight and $\lambda_{\text {syn }}$ is a scaling factor calculated so that a synaptic weight of $1 \mathrm{mV}$ produces postsynaptic potentials (PSPs) of peak size $1 \mathrm{mV}$. Note that $g_{\text {syn }}$ is in units of volt, i.e., the membrane resistance is implicitly included in the variable.

Spike-timing-dependent plasticity. The weights of excitatory synapses evolve according to an additive spike-timing-dependent plasticity (STDP) rule (Markram et al., 1997; Bi and Poo, 1998; Song and Abbott, 2001). Each pair of presynaptic and postsynaptic spikes produces an increase $\delta w$ in weight:

$$
\delta w=\left\{\begin{array}{l}
A_{\mathrm{pot}} e^{-\frac{\left|\delta_{t}\right|}{\tau_{\mathrm{pot}}}} \text { if } \delta t=t_{\mathrm{post}}-t_{\mathrm{pre}}>0 \\
-A_{\mathrm{dep}} e^{-\frac{\left|\delta_{t}\right|}{\tau_{\text {dep }}}} \text { if } \delta t=t_{\mathrm{post}}-t_{\mathrm{pre}} \leq 0
\end{array} .\right.
$$

In addition, weights are clipped between $0 \mathrm{mV}$ and $w_{\max }=2 \mathrm{mV}$. We set $A_{\text {dep }}=1.2 A_{\text {pot }}=\varepsilon w_{\text {max }}$, with $\varepsilon=1 / 100$, and $\tau_{\text {pot }}=\tau_{\text {dep }}=20 \mathrm{~ms}$.

In recurrent network simulations, inhibitory to excitatory synapses are also plastic, following the results of Vogels et al. (2011). For every neuron $k$, a synaptic trace $x_{\mathrm{k}}$ is assigned such that at each spike $x_{\mathrm{k}} \leftarrow x_{\mathrm{k}}+1$, and otherwise $\tau_{\text {pot }} \frac{d x_{k}}{d t}=-x_{k}$. Synaptic weight between presynaptic neuron $i$ and postsynaptic neuron $j$ is updated as follows:

$$
\delta w=\left\{\begin{array}{c}
\eta\left(x_{j}-\gamma\right) \text { if presynaptic spike } \\
\eta x_{i} \text { if presynaptic spike }
\end{array},\right.
$$

where $\eta$ is the learning rate (here $\left.\eta=w_{\max } / 100\right)$ and $\gamma=-2 \rho_{0} \tau_{\text {pot }}$, where $\rho_{0}=2 \mathrm{~Hz}$ is the target firing rate of the network.
In Figure 9, we show that the results are valid for several learning rules. We consider the case of binary synapses, whose probability of switch at each post or presynaptic spike [respectively, $\zeta^{\text {post }}(t)$ and $\zeta^{\text {pre }}(t)$ ] is a function of the traces $A_{\text {post }}(t)$ and $A_{\text {pre }}(t)$, defined by $A_{\text {pre/post }} \leftarrow A_{\text {pre/post }}+A_{\text {pot/dep }}$ at each spike, and otherwise $\tau_{\text {pot/dep }} \frac{A_{\text {pre/post }}}{d t}=-A_{\text {pre/post }}$. More specifically, we have the following:

$$
w=\left\{\begin{array}{l}
0 \text { at post spike with probability } \zeta^{\text {post }}(t) \\
w_{\max } \text { at pre spike with probability } \zeta^{\text {pre }}(t)
\end{array} .\right.
$$

To keep the same mean synaptic modifications as previously, we used $\zeta^{\text {post }}(t)=A_{\text {post }}(t)\langle w\rangle / A_{\text {pot }}$ and $\zeta^{\text {pre }}(t)=A_{\text {pre }}(t)\left(w_{\max }-\langle w\rangle\right) / A_{\text {dep }}$, where $\langle w\rangle$ is the mean of the synaptic weight obtained after convergence to the equilibrium (Fig. 1). The triplet learning rule is implemented as in the work of Pfister and Gerstner (2006), with the simplified set of parameters.

Feedforward simulations. Each neuron receives $N_{\mathrm{e}}=8000$ excitatory inputs and $N_{\mathrm{i}}=2000$ inhibitory inputs, firing according to Poisson processes with firing rate $F_{\mathrm{e}}=F_{\mathrm{i}}=1 \mathrm{~Hz}$ (Fig. $1 A$ ). The initial excitatory weights are uniformly drawn between 0 and $w_{\max }$, and inhibitory weights are set such that $w_{\mathrm{i}}=-1 \mathrm{mV}$ (the results were unchanged with heterogeneous weights). Simulations run for $30 \mathrm{~h}$ (biological time) before the presentation of patterns, so as to reach equilibrum. Repeated patterns are presented by pregenerating a single 500 -ms-long spatiotemporal pattern of spike trains (identical statistical properties) and presenting it every $2 \mathrm{~s}$. In Figure 7, $A$ and $B$, we added a normally distributed jitter to all the spike times of the pattern, independent between all spikes and between presentations. In Figure 7, $C$ and $D$, we added Gaussian white noise to the right side of the membrane equation: $\sigma_{\mathrm{v}}=\sqrt{2 \tau_{m} \xi}(t)$.

Reliability measure. We measured response reliability with the same method as in the work of Yao et al. (2007). For each neuron, spike trains were binned with a $50 \mathrm{~ms}$ time bin, and we reported the correlation coefficient between each repeat $r_{\mathrm{i}}$ and the average of its nearest four repeats. Using a smaller time bin $(5 \mathrm{~ms})$ did not change the results.

Recurrent simulations. We used a random balanced network (van Vreeswijk and Sompolinsky, 1996) composed of 8000 excitatory neurons and 2000 inhibitory neurons (see Fig. 8A). Every neuron in the network is connected to $20 \%$ of the others, with delays drawn from a uniform distribution between 0 and $5 \mathrm{~ms}$. Within the network, all the excitatory connections are plastic, governed by a classical STDP rule (see Eq. 1). All inhibitory to excitatory connections are also plastic (see corresponding sections; Vogels et al., 2011) to ensure the stability of the balanced regime. All excitatory neurons within the network are stimulated with $N_{\text {ext }}=8000$ external excitatory inputs, as in the feedforward scenario. External inputs project to those neurons with connection probability $20 \%$ and uniformly randomized delays, and those connections are also plastic. Initial synaptic weights for recurrent connections are drawn from Gaussian distributions such that $\left\langle w_{\mathrm{e}}\right\rangle=0.2 \mathrm{mV},\left\langle w_{\mathrm{i}}\right\rangle=-1 \mathrm{mV}$. SDs are chosen to be one-fourth of the means. The initial strength of the external synapses is also drawn from a Gaussian distribution such that $\left\langle w_{\text {ext }}\right\rangle=\left\langle w_{\mathrm{e}}\right\rangle$. To sustain the asynchronous activity, a constant input of $V_{\text {thresh }}-V_{\text {rest }}=15$ $\mathrm{mV}$ is injected into all neurons as in the study by Vogels et al. (2011).

Theory. To calculate the $V_{\mathrm{m}}$ distribution, we neglect the contribution of spikes and use Campbell's theorems, which give the mean and variance of a shot noise (corresponding to sums of PSPs with Poisson statistics):

$$
\begin{gathered}
\left\langle V_{\mathrm{m}}\right\rangle=F_{e} N_{e}\left\langle\int E P S P\right\rangle+F_{i} N_{i}\left\langle\int I P S P\right\rangle+V_{\text {rest }}, \\
\sigma^{2}\left(V_{\mathrm{m}}\right)=F_{e} N_{e}\left\langle\int E P S P^{2}\right\rangle+F_{i} N_{i}\left\langle\int I P S P^{2}\right\rangle,
\end{gathered}
$$

where averages are over synapses. PSPs of the same type vary only by their synaptic strength (defined as the peak value): EPSP $=w_{\mathrm{e}}$ EPSP $^{*}$ and IPSP $=w_{\mathrm{i}} \mathrm{IPSP}^{*}$, where:

$$
P S P(t)=\lambda_{s} \frac{\tau_{s}}{\tau_{m}-\tau_{s}}\left(e^{-t / \tau_{m}}-e^{-t / \tau_{s}}\right)
$$


A

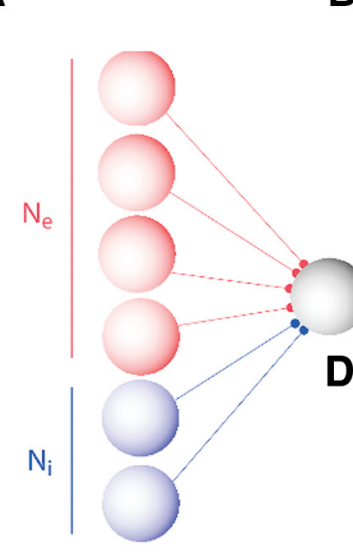

E

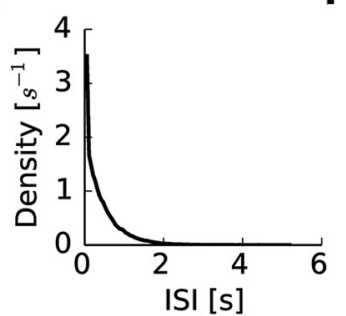

$\mathbf{F}$
B

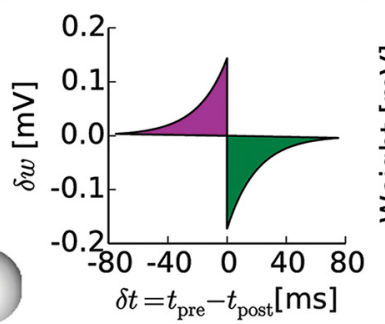

C

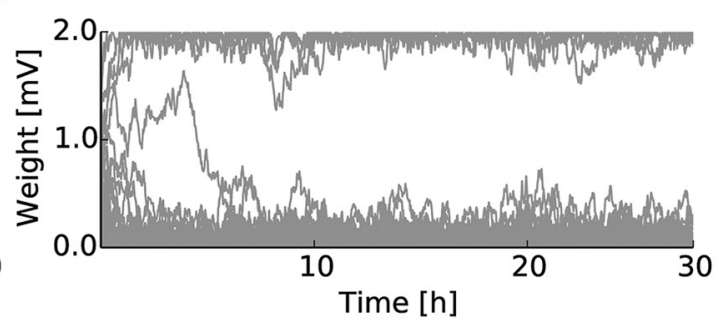

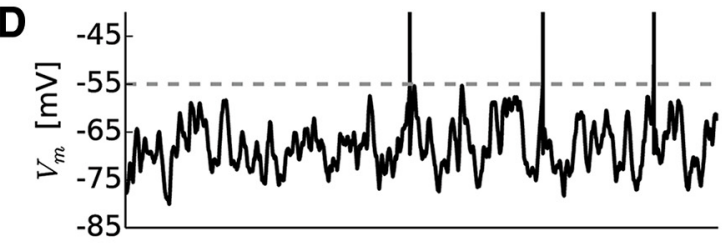
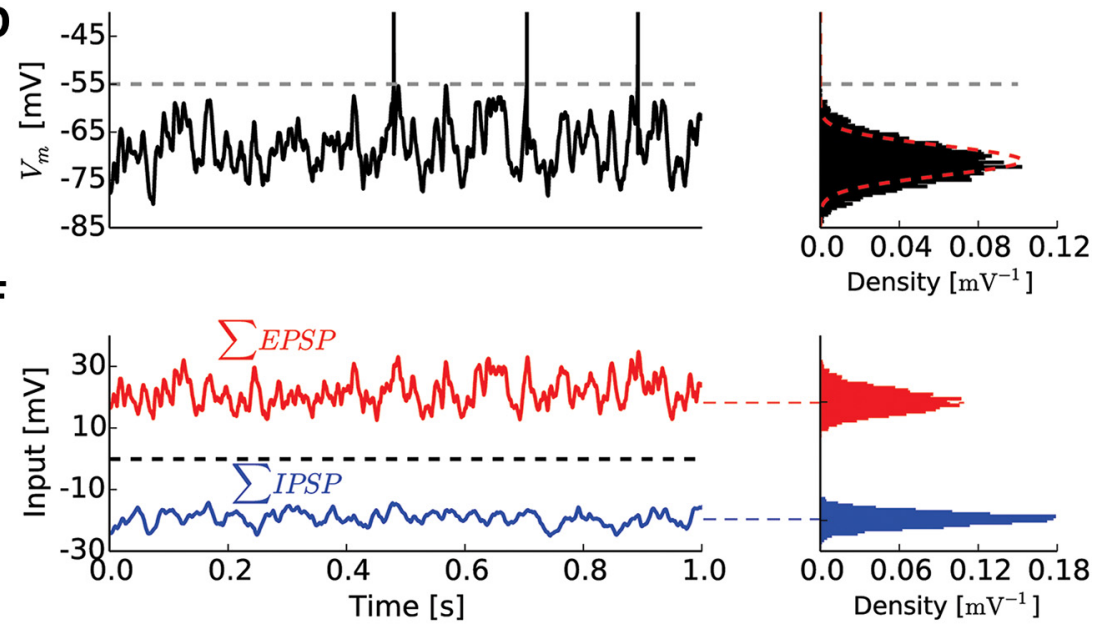

Figure 1. The balanced regime. $A$, A neuron model receives random spike trains from $N_{\mathrm{e}}=8000$ excitatory and $N_{\mathrm{i}}=2000$ inhibitory inputs. $\boldsymbol{B}$, Synaptic modification for one pair of presynaptic and postsynaptic spikes as a function of their relative timing. C, Evolution of 50 randomly chosen synaptic weights over $30 \mathrm{~h}$ (biological time). $\boldsymbol{D}$, Left, Sample voltage trace at equilibrium (dashed line, firing threshold). Right, $V_{m}$ distribution (dashed curve, theoretical prediction). $\boldsymbol{E}$, Distribution of the interspike intervals (ISI). $\boldsymbol{F}$, Sample current traces at equilibrium for total excitation (red) and total inhibition (blue), with distributions shown on the right.

where $\tau_{s}$ is the synaptic time constant and $\lambda_{s}$ is the normalization factor (defined above). We define $\alpha_{\mathrm{e}}=\int \operatorname{EPSP}(s) d s=\lambda_{\mathrm{e}} \tau_{\mathrm{e}}$ and $\beta_{e}=$ $\int \operatorname{EPSP}(s)^{2} d s=\lambda_{e}^{2} \frac{\tau_{e}^{2}}{2\left(\tau_{m}+\tau_{e}\right)}$, and accordingly for $\alpha_{\mathrm{i}}$ and $\beta_{i}$.

To estimate $\sigma^{2}\left(V_{\mathrm{m}}\right)$ at equilibrium in a neuron with synapses governed by STDP, we assume that (1) all excitatory synapses are either weak $\left(w_{\mathrm{e}}=0 \mathrm{mV}\right)$ or maximally potentiated $\left(w_{\mathrm{e}}=w_{\max }\right)$ and (2) excitation and inhibition are balanced, so that $\left\langle V_{\mathrm{m}}\right\rangle=V_{\text {rest }}$. This latter approximation, which we call the balance equation (using Eq. 4), is valid when both excitation and inhibition are strong. Denoting $p$ the proportion of active (nonweak) synapses, the balance equation reads as follows:

$$
F_{e} N_{e} p w_{\max } \alpha_{e}+F_{i} N_{i} w_{i} \alpha_{i}=0 .
$$

The substitution in Equation 5 gives the following:

$$
\sigma^{2}\left(V_{m}\right)=F_{i} N_{i} w_{i}\left(w_{i} \beta i-p w_{\max } \beta_{e} \alpha_{i} / \alpha_{e}\right) .
$$

This theoretical prediction is displayed in Figure $1 D$ and used in Figure $3 C$. In Figure $3 A$, we consider a neuron with uniform excitatory and inhibitory synapses (a single pair of values for $w_{\mathrm{e}}$ and $w_{\mathrm{i}}$ ), and we calculate $w_{\mathrm{e}}$ and $w_{\mathrm{i}}$ so that $\left\langle V_{\mathrm{m}}\right\rangle=V_{\text {rest }}$ and $\sigma^{2}\left(V_{\mathrm{m}}\right)$ is a given value. Here the balance equation reads as follows:

$$
F_{e} N_{e} w_{e} \alpha_{e}+F_{i} N_{i} w_{i} \alpha_{i}=0 .
$$

The substitution in Equation 5 gives the following:

$$
\begin{gathered}
\sigma^{2}\left(V_{m}\right)=\frac{\beta_{e} F_{i}^{2} N_{i}^{2} \alpha_{i}^{2} w_{i}^{2}}{F_{e} N_{e} \alpha_{e}^{2}}+F_{i} N_{i} \beta_{i} w_{i}^{2} \\
=F_{i} N_{i} w_{i}^{2}\left(\beta_{i}+\beta_{e} \frac{F_{i} N_{i} \alpha_{i}^{2}}{F_{e} N_{e} \alpha_{e}^{2}}\right) .
\end{gathered}
$$

This equation gives $w_{\mathrm{i}}$ as a function of $\sigma^{2}\left(V_{\mathrm{m}}\right)$, so that

$$
w_{i}^{2}=\frac{\sigma^{2}\left(V_{m}\right)}{F_{i} N_{i}}\left(\beta_{i}+\beta_{e} \frac{F_{i} N_{i} \alpha_{i}^{2}}{F_{e} N_{e} \alpha_{e}^{2}}\right)^{-1} .
$$

Once $w_{\mathrm{i}}$ is obtained, $w_{\mathrm{e}}$ is deduced using the balanced Equation 9, and these values are used in Figure $3 A$. Note that both $w_{\mathrm{i}}$ and $w_{\mathrm{e}}$ scale as $1 / \sqrt{N}$.

Simulator. All simulations were performed using the Brian simulator version 2 (Goodman and Brette, 2009) with a fixed time step of $0.1 \mathrm{~ms}$.

\section{Results}

\section{The balanced regime}

Cortical neurons receive inputs from about 10,000 synapses (Braitenberg and Schütz, 1991), resulting in an intense bombardment of synaptic activity in vivo (Destexhe et al., 2003). Approximately $80 \%$ of those synapses are excitatory, and it is believed that the large excitatory drive is balanced by a similarly large inhibitory drive (Okun and Lampl, 2008). This "balanced regime" (van Vreeswijk and Sompolinsky, 1996; Brunel, 2000; Renart et al., 2010) has several characteristic features observed in vivo. First, the membrane potential $\left(V_{\mathrm{m}}\right)$ distribution peaks below threshold [Rossant et al. (2011), their Fig. 7]. Second, neurons fire irregularly when excitation transiently exceeds inhibition (Softky and Koch, 1993; Shadlen and Newsome, 1998). Third, their firing rates are relatively low (Koulakov et al., 2009; Bruno, 2011).

As shown previously (Song and Abbott, 2001), these features can be reproduced by a simple neuron model undergoing STDP (Gerstner et al., 1996; Markram et al., 1997; Bi and Poo, 1998) where long-term depression is larger than long-term potentiation. In Figure 1, we simulated an integrate-and-fire model with $N_{\mathrm{e}}=8000$ excitatory inputs and $N_{\mathrm{i}}=2000$ inhibitory inputs (Fig. 
A

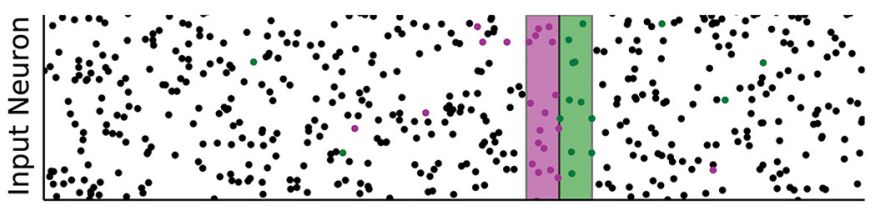

B

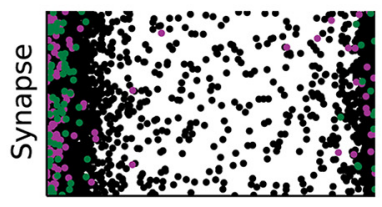

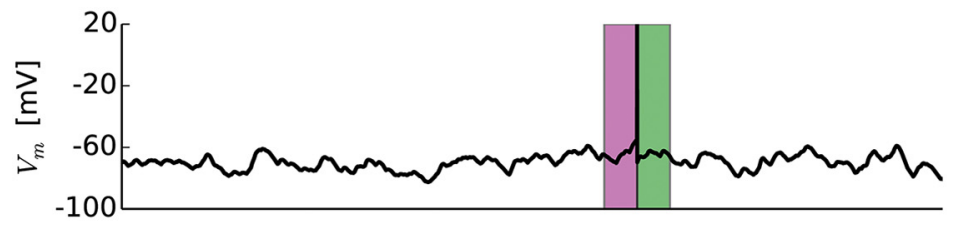

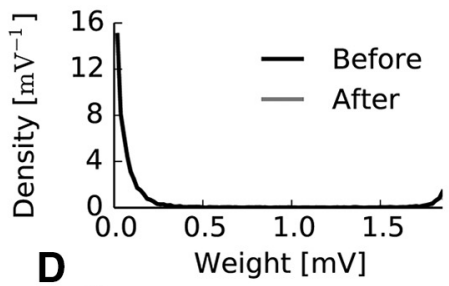

C

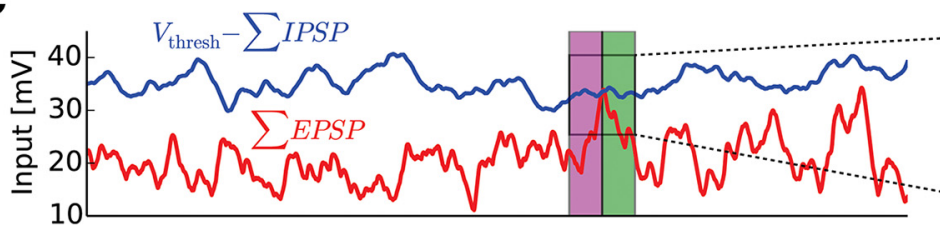

\section{E}
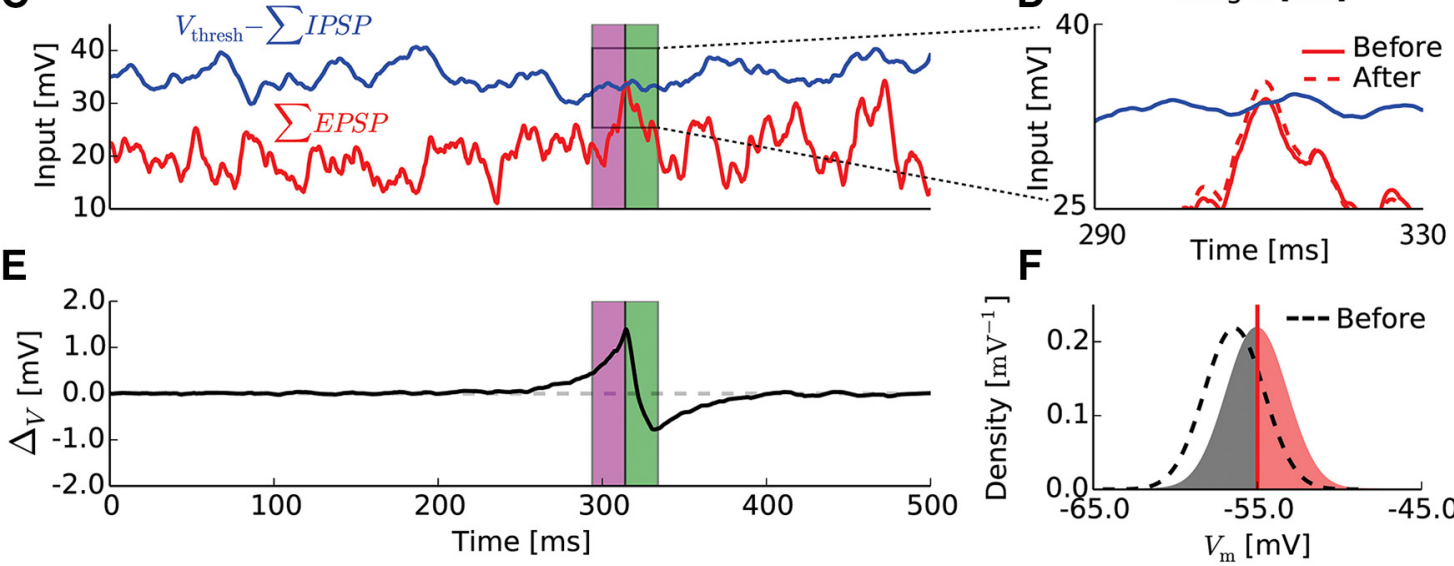

2. Synaptic plasticity induced by a single spike in the balanced regime. A, Top, The neuron receives random spike trains from 8000 excitatory inputs (1000 shown) and 2000 inhibitory inputs (not shown). Bottom, The neuron fires irregularly in response to input fluctuations. Synapses receiving a presynaptic spike shortly before a postsynaptic spike $\left(<\tau_{\text {pot }}=20 \mathrm{~ms}\right.$, purple) are potentiated; those with a presynaptic spike arriving shortly after $\left(<\tau_{\text {dep }}=20 \mathrm{~ms}\right.$, green) are depressed. $\boldsymbol{B}$, The distribution of weights at equilibrium is bimodal, with many weak synapses. A single spike modifies both weak and strong synapses (top, colored dots) and produces no change in the weight distribution (bottom). $C$, Spikes are produced when summed EPSPs (red) are greater than threshold minus summed IPSPs (blue). D, Zoom on a $40 \mathrm{~ms}$ window around the spike. After synaptic modifications (dashed line), the same excitatory pattern produces a larger excitatory drive near spike time. $\boldsymbol{E}$, Change $\Delta_{V}$ in excitatory drive attributable to synaptic modifications between two successive presentations of the same excitatory input pattern (difference between the summed EPSPs before and after presentation). $\boldsymbol{F}$, Distribution of $V_{\mathrm{m}}$ at the time of the spike $(t=310 \mathrm{~ms})$, before (dashed line) and after (filled area) synaptic modifications, for the same excitatory input pattern (here the source of variability is random inhibition). The area on the right of the threshold (red line) represents the firing probability and increases from $22 \%$ to $50 \%$.

$1 A$ ), randomly firing at $1 \mathrm{~Hz}$ with no correlations (see Discussion for the impact of correlations). The excitatory synapses undergo STDP (Fig. $1 B$ ) with a maximum PSP of $2 \mathrm{mV}$ (time constant $\tau_{\mathrm{e}}=3 \mathrm{~ms}$ ), whereas inhibitory synapses were not plastic (PSP size, $-0.5 \mathrm{mV}$; time constant $\tau_{\mathrm{i}}=10 \mathrm{~ms}$ ); we examine inhibitory plasticity in a later part. Results are shown at equilibrium, after $30 \mathrm{~h}$ of biological time (Fig. $1 C$ ). The neuron then fires irregularly $(\langle\mathrm{CV}\rangle \simeq 1)$ at a low firing rate $(F=2.17 \mathrm{~Hz}$; Fig. $1 D, E)$. The $V_{\mathrm{m}}$ distribution is approximately Gaussian and peaks near $-71 \mathrm{mV}$, $\simeq 1 \mathrm{mV}$ below the resting potential (Fig. $1 D$ ). The excitatory drive is large, corresponding to a mean depolarization of $22 \mathrm{mV}( \pm 6.7$ $\mathrm{mV}$ ) in the absence of inhibition, but is matched by a large amount of inhibition so that the mean $V_{\mathrm{m}}$ is well below threshold (Fig. $1 F$ ). Note that we modeled synaptic inputs as currents rather than conductances so that we can easily compare with analytical calculations (Fig. $1 D$, dashed line shows the theoretical prediction for the $V_{\mathrm{m}}$ distribution), but the same basic properties would hold with synaptic conductances (El Boustani and Destexhe, 2009), where the balance equation is the equality of mean excitatory and inhibitory currents at mean $V_{\mathrm{m}}$ (Richardson and Gerstner, 2005).
Synaptic plasticity induced by a single spike

We now examine the effect of a single output spike on synaptic modifications, when the neuron receives random inputs $\left(F_{\mathrm{e}}=\right.$ $F_{\mathrm{i}}=1 \mathrm{~Hz}$; Fig. 2A). With a LTP window of size $\tau_{\text {pot }}=20 \mathrm{~ms}$, approximately $N_{\text {syn }}=\tau_{\text {pot }} F_{\mathrm{e}} N_{\mathrm{e}}=160$ synapses are potentiated, on average, and a similar number are depressed. This is a small fraction of the 8000 excitatory synapses, and each synaptic modification represents only $\varepsilon=1 \%$ of the maximum PSP size (i.e., $\delta w=20 \mu \mathrm{V}$ ), in accordance with experimental observations (Bi and Poo, 1998; Wang et al., 2005), so that the distribution of synaptic weights is essentially unchanged by this single spike (Fig. $2 B)$. However, the total synaptic modification is a sizable fraction of maximum PSP size $(160 \times 1 \%=160 \%)$. If the same pattern of excitatory inputs were presented again, this would result in a corresponding change in the excitatory-inhibitory balance at the time of the previous spike that triggered LTP (Fig. 2C-E), where $\Delta_{\mathrm{V}}$ is the total change in depolarization attributable to the plastic changes. Such a misbalance has a strong effect on firing probability in the balanced regime (Rossant et al., 2011). Figure $2 F$ shows the probability distribution of $V_{\mathrm{m}}$ at the time of the output spike for the particular pattern of excitatory inputs shown in Figure $2 \mathrm{~A}$ 

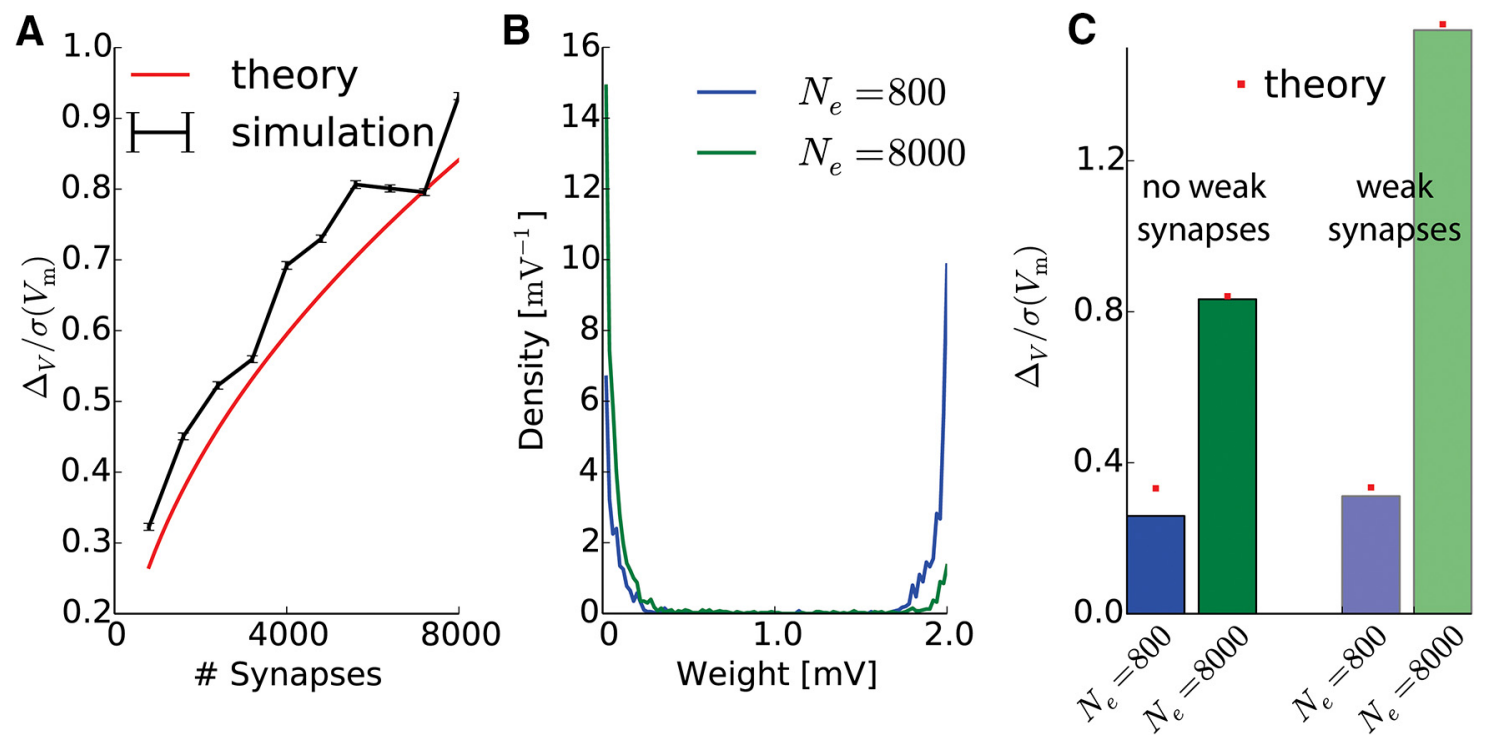

Figure 3. Theoretical impact of a single spike on total synaptic potentiation. $A$, Total synaptic potentiation $\left(\Delta_{\mathrm{V}}\right.$, as shown in Fig. $\left.2 E\right)$ caused by a single output spike, relative to the $V_{\mathrm{m}}\left[\sigma\left(V_{\mathrm{m}}\right)\right]$, as a function of the number of excitatory synapses. $\boldsymbol{B}$, Distribution of synaptic weights at equilibrium for 800 (blue) and 8000 (green) plastic excitatory synapses onto a single neuron. $\boldsymbol{C}$, Total synaptic potentiation $\Delta_{V}$ relative to $\sigma\left(V_{m}\right)$ for 800 and 8000 synapses, without weak synapses (left) and with weak synapses (right).

(here inhibition is the source of variability), before and after synaptic modification. The probability that the neuron fires in response to the excitatory pattern (red area) goes from $22 \%$ to $50 \%$ because of the synaptic changes. Thus, plasticity induced by a single output spike more than doubles the probability that the neuron fires to the same input.

This phenomenon can be explained theoretically by a leverage effect caused by the large number of synapses that contribute to relatively small fluctuations in $V_{\mathrm{m}}$ in the balanced regime (van Vreeswijk and Sompolinsky, 1996). Assuming inputs are independent, the standard deviation of the excitatory drive (measured as current, conductance, or total depolarization) scales as $\sqrt{N_{e}}$ (see Materials and Methods for detailed calculations). In contrast, total synaptic potentiation induced by a single spike is $\varepsilon N_{\mathrm{e}} F_{\mathrm{e}} \tau_{\text {pot }} \max (\mathrm{EPSP})$ and therefore scales as $N_{\mathrm{e}}$. Thus, the relative misbalance (the difference between excitatory and inhibitory drives) induced by a single spike scales as $\sqrt{N_{e}}$. For cortical neurons that have a large number of excitatory synapses, this produces a strong leverage effect. That is, the balance condition multiplies the relative effect of a synaptic modification by $\sqrt{N_{e}}$ so that small individual synaptic modifications result in a substantial increase in firing probability.

To demonstrate this leverage effect, we simulated an integrate-and-fire model receiving random excitatory and inhibitory inputs $\left(F_{\mathrm{e}}=F_{\mathrm{i}}=1 \mathrm{~Hz}\right)$ through synapses with fixed weights, in a 4:1 ratio (Fig. 3). The synaptic weights for excitation $w_{\mathrm{e}}$ and inhibition $w_{\mathrm{i}}$ were adjusted so that the mean $V_{\mathrm{m}}$ equals the resting potential (imposing a linear relationship between the weights $w_{\mathrm{e}}$ and $w_{\mathrm{i}}$; see Materials and Methods), and the $\operatorname{SD} \sigma\left(V_{\mathrm{m}}\right)$ is $5.2 \mathrm{mV}$ (imposing an affine relationship between $w_{\mathrm{e}}^{2}$ and $w_{\mathrm{i}}^{2}$ ). The value of $5.2 \mathrm{mV}$ is arbitrary, similar to the value measured in the equilibrium obtained in Figure 1. Therefore, to keep the SD of the voltage constant, weights had to be scaled as $1 / \sqrt{N}$ (see Materials and Methods for analytical values). We then measured the change in total depolarization $\Delta_{V}=\Sigma_{\mathrm{i}} \delta w_{\mathrm{i}}$ attributable to STDP at firing times by stimulating the neuron again with the same input pattern, as done in Figure $2 E$ (bottom). Figure $3 A$ shows the mean total increase for different numbers of synapses in units of $\sigma\left(V_{\mathrm{m}}\right)$. The results are well matched by theoretical predictions and show that the total modification induced by a single spike is a sizable fraction of $\sigma\left(V_{\mathrm{m}}\right)$.

There is an additional leverage effect in the situation shown in Figure 2, coming from the large proportion of weak synapses ( $90 \%$ of synaptic weights are smaller than half the maximum; Fig. $2 B)$. This is the result of the STDP mechanism we chose, which produces bimodal distributions of weights (van Rossum et al., 2000; Gütig et al., 2003), and this is consistent with the fact that neurons, in particular hippocampal cells, are known to have a large fraction of weak synapses (Isaac et al., 1995; Rumpel et al., 1998). Weak synapses do not contribute to the $V_{\mathrm{m}}$ fluctuations, but they can, in principle, be potentiated (Fig. $2 A, B$, green dots). That is, if $p$ is the proportion of nonweak synapses, then $\sigma\left(V_{\mathrm{m}}\right)$ is proportional to $\sqrt{p N_{e}}$ and the synaptic potentiation induced by a single spike is proportional to $N_{\mathrm{e}}$. Thus, in units of $\sigma\left(V_{\mathrm{m}}\right)$, total synaptic potentiation is proportional to $\sqrt{N_{e} / p}$. To illustrate this effect, we measured the total depolarization attributable to STDP at firing times as previously, but with excitatory weights resulting from a long simulation with STDP (as in Fig. 2). The distribution of these weights is shown in Figure $3 B$ for two different numbers of excitatory synapses (inhibitory synapses are set as previously; Fig. 2). The proportion of weak synapses can be theoretically predicted using the balance equation (see Materials and Methods), and therefore total depolarization attributable to STDP can also be predicted. Numerical results agree with theory and show that the presence of weak synapses indeed introduces an additional leverage effect (Fig. 3C).

\section{Fast learning, slower forgetting}

Because of these two leverage effects, neurons can learn to respond to a repeated pattern, as shown in Figure 4. We simulated the same model as in Figure 2, and we embedded a repeated 500 $\mathrm{ms}$ pattern of excitatory inputs into the random stream of spikes, with the same statistics (independent Poisson inputs at $F_{\mathrm{e}}=1 \mathrm{~Hz}$; Fig. $4 A$ ). The pattern was repeated every $2 \mathrm{~s}$. The only difference between the pattern and background activity is that it is repeated. The output of 5000 independent neurons responding to the same 
pattern is shown in Figure $4 B$. After just 10 presentations, the neurons respond much more to the repeated pattern than to background activity ( $\sim 8 \mathrm{~Hz}$ vs $2 \mathrm{~Hz}$; Fig. $4 B$ ), even though synaptic weights have changed very little (Fig. $4 C, D$ ). Figure $4 E$ shows the response of two sample neurons to the repeated pattern over several successive presentations. The firing rate appears to increase by inserting spikes at precise times, and response reliability (see Materials and Methods) also tends to increase (Fig. 4F). This observation agrees with in vivo experiments in which response reliability was seen to increase in response to repeated presentations of natural stimulus (Yao et al., 2007).

In contrast with learning, forgetting is not impacted by the leverage effects discussed above and therefore is much slower. In Figure $5 A$, we presented the model with 10 repetitions of the same excitatory input pattern, presented random inputs for $2 \mathrm{~min}$, and presented the repeated pattern again. After the $2 \mathrm{~min}$ pause, neurons respond to the pattern with about the same firing rate as they did just before the pause. This observation mimics experimental observations in vivo, where enhanced cortical responses to natural stimulus persisted after the presentation of unrelated stimuli (Yao et al., 2007; Bermudez Contreras et al., 2013). Why is forgetting much slower than learning? This is simply because erasing a memory requires modifying the same synapses that have been potentiated, but a given synapse has a low probability of being modified by a random input. For a given output spike, the probability that an excitatory input spike falls in the temporal window of depression $\left(\tau_{\text {dep }}=20 \mathrm{~ms}\right.$ here $)$ is $\tau_{\text {dep }} F_{\mathrm{e}}=$ 0.02 . If the neuron spikes at a rate $F_{\text {post }}=$

$2 \mathrm{~Hz}$, then a given synapse is depressed randomly at rate $\tau_{\mathrm{dep}} F_{\mathrm{e}} F_{\text {post }}=0.04 \mathrm{~Hz}$, i.e., once every $25 \mathrm{~s}$. In addition, that synapse may be depressed, but it may also be potentiated. The net average modification is the integral of the STDP function (Fig. $1 B)$, so the rate of modification is $F_{\mathrm{e}} F_{\text {post }}\left(A_{\text {pot }} \tau_{\text {pot }}-A_{\text {dep }} \tau_{\text {dep }}\right.$, where $A_{\text {pot }}$ is the peak LTP and $A_{\text {dep }}$ is the peak LTD), which must be compared with a previous potentiation of approximately $A_{\text {pot. }}$. Thus, the relative rate of synaptic decay is $F_{\mathrm{e}} F_{\text {post }}\left(\tau_{\text {pot }}-\tau_{\mathrm{dep}} A_{\mathrm{dep}} /\right.$ $\left.A_{\text {pot }}\right)=0.8 \% / s$ here. This decay corresponds to a retention time of $\sim 2 \mathrm{~min}$. In Figure $5 B$, we show the firing rate of the neurons just after the pause as a function of pause duration for different initial numbers of presentations of the pattern. It appears that the retention time, defined as the time when the response to the stimulus has decayed by $50 \%$, is of the order of 15 min (Fig. $5 C$ ). The theoretical prediction underestimates the retention time because the calculation implicitly assumes that inputs and outputs are independent, which is only true for weak synapses. As can be seen in Figure $5 D$, the average modification for the weight change (solid red curve) matches the theoretical prediction (dashed line) for weak synapses, but not for strong synapses. For the latter ones, weights tend to increase because they can cause output spikes and thus induce LTP, which increases the retention time calculated above. We note that although forgetting is slower than learning, the mechanism we describe explains the retention of memories over minutes or tens of minutes, but not over days or weeks. We assume that longer time scales involve additional consolidation mechanisms (see Discussion).

\section{Interference between patterns}

What happens when two patterns are presented? In Figure 6, we consider two different scenarios. In the first scenario, two independent patterns are repeatedly presented in an interleaved fashion, 10 times each (Fig. 6A). Neurons fire increasingly to both patterns (Fig. $6 B$ ). When the two patterns are presented again after a $110 \mathrm{~s}$ pause, neurons fire again at the same rate as in the last presentation before the pause. In the second scenario, a pattern is repeatedly presented 10 times, then a second pattern is presented 10 times, and the first pattern is presented again 10 times (Fig. $6 C)$. It appears that there is no interference between the two patterns (Fig. 6D). That is, when the second pattern is presented, neurons fire increasingly as they did for the first pat- 
A

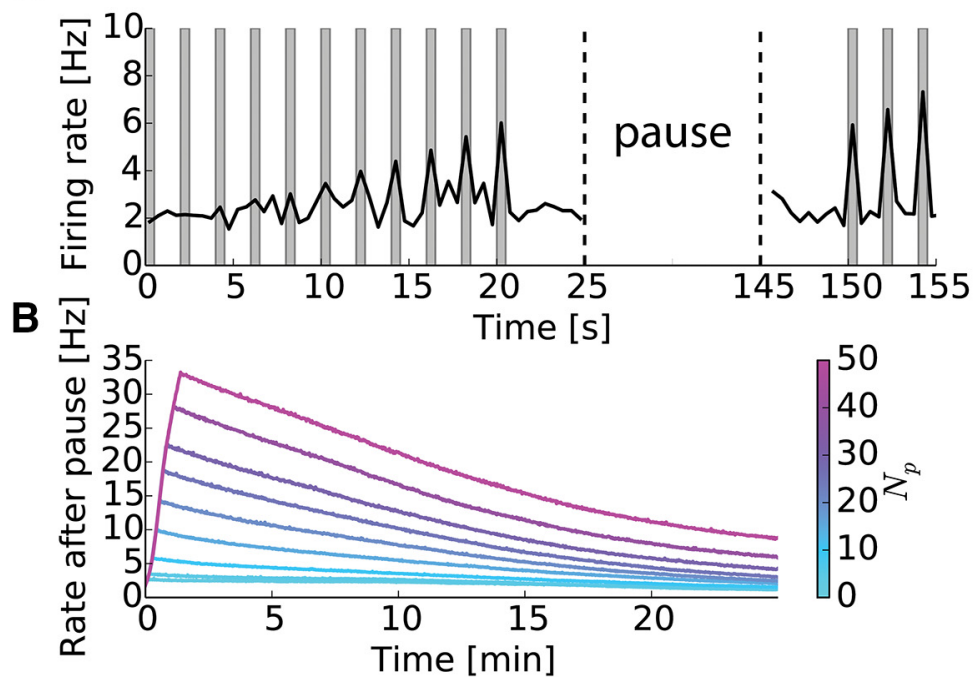

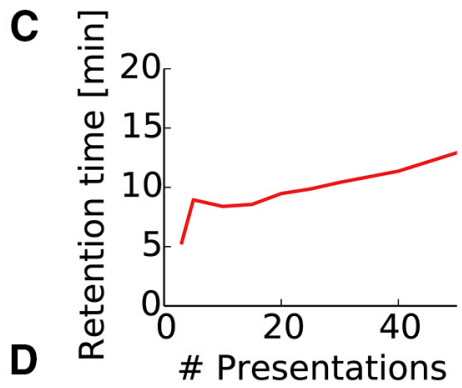

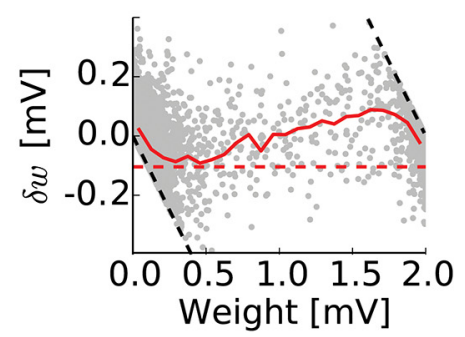

Figure 5. Memory retention after learning. $\boldsymbol{A}$, The same pattern is presented 10 times, once every $2 \mathrm{~s}$ (as in Fig. 4), resulting in a pattern-specific rate increase in postsynaptic neurons. The presentation is paused for $130 \mathrm{~s}$, while neurons receive random inputs, and then the pattern repetition resumes. Pattern selectivity has not been altered by the pause. $\boldsymbol{B}$, Postsynaptic rate after the pause as a function of pause duration for various numbers of initial presentations. $\boldsymbol{C}$, Half-life of the response to the repeated pattern as a function of the number of initial presentations. $\boldsymbol{D}$, Modification of synaptic weight after $10 \mathrm{~min}$ of random input as a function of the initial weight (solid red curve, average). The dashed red line is the prediction for uncorrelated input/output spikes. The dashed black lines correspond to the constraints imposed by weight boundaries ( 0 and $2 \mathrm{mV}$ ).
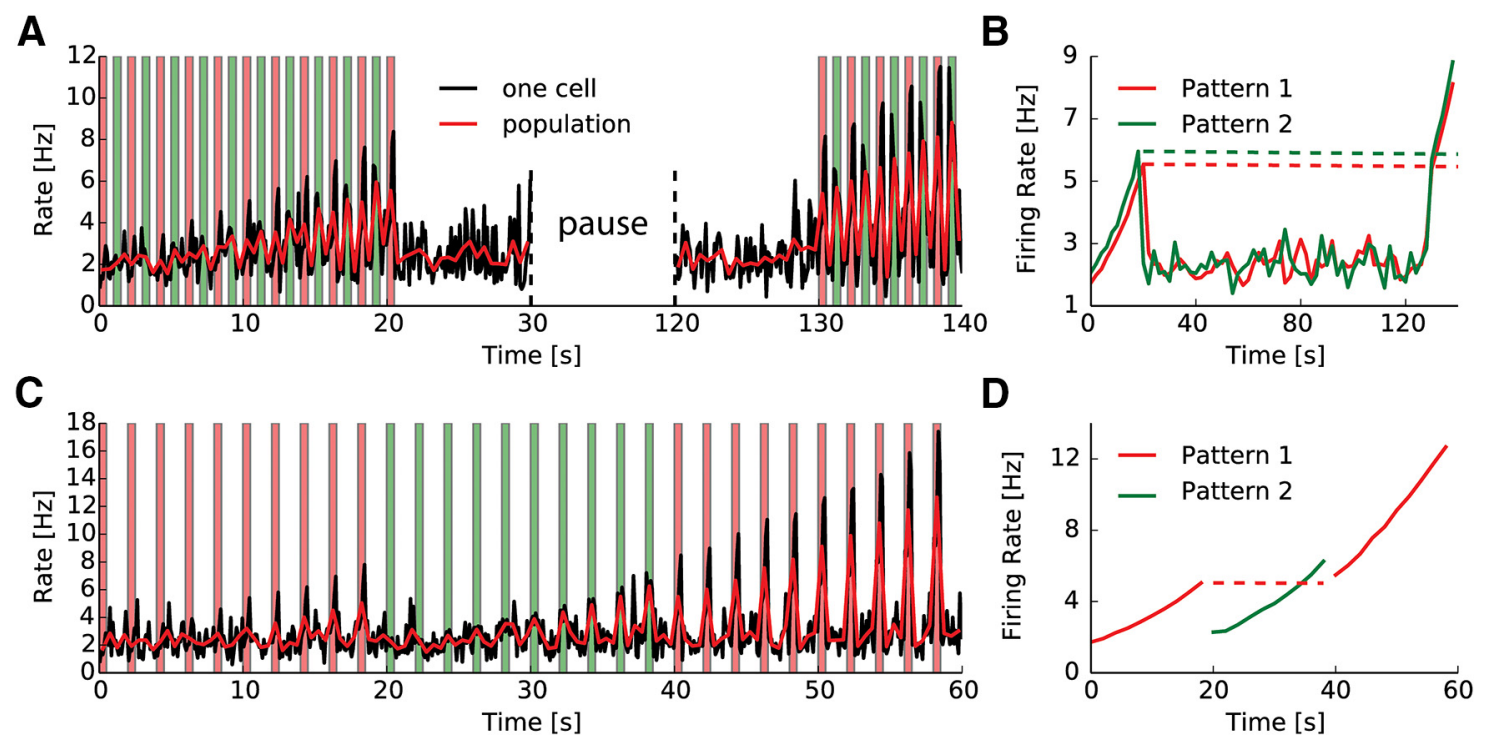

Figure 6. Interference between patterns. $\boldsymbol{A}$, Two patterns are presented 10 times each, interleaved and once every $2 \mathrm{~s}$ (as in Fig. 4), resulting in a pattern-specific rate increase in postsynaptic neurons. The presentations are paused for $110 \mathrm{~s}$, while neurons receive random inputs, and then the pattern repetitions resume. $\boldsymbol{B}$, Firing rate during the pattern presentations, for those two patterns. Dashed lines represent the theoretical decay time. C, A first pattern (red) is presented 10 times (as in Fig. 4), followed by a second pattern (green), and then the first pattern is presented 10 times again. As can be seen, the presentation of the second pattern does not interfere with the first learning. $\boldsymbol{D}$, Same as $\boldsymbol{B}$ for the simulation shown in $\boldsymbol{C}$.

tern; when the first pattern is presented again, neurons start firing at about the same rate as in the last presentation of that pattern. This lack of interference is expected because an output spike triggers potentiation for a small proportion of synapses (on the order of $F_{\mathrm{e}} \tau_{\text {pot }}=0.5 \%$ ), and therefore there should be little overlap in the potentiated synapses between two independent patterns.

\section{Requirements for fast learning}

The effect we have shown relies mainly on the neuron being in the balanced regime, where spikes are caused by transient increases in excitation relative to inhibition (or decrease of inhibition), and on the leverage effect because of the large number of synapses.
Another implicit requirement is that the precise spike times of the stimulus are reproducible. Indeed, the synaptic modifications caused by a single spike can only contribute to an increased response to the stimulus if the same synapses are simultaneously coactivated. More precisely, the relative spike times or at least the synchrony events must be reproducible. This is shown in Figure 7, $A$ and $B$, where we introduced random jitter in the input spike times of our model. When the jitter is small, the firing rate in response to the stimulus increases with the number of presentations, but the effect vanishes when the jitter exceeds the membrane time constant $\left(\tau_{\mathrm{m}}=\right.$ $5 \mathrm{~ms}$ here). Synaptic failures have no impact on spike timing and, therefore, may quantitatively decrease the rate of learning but have no consequence on the basic leverage effect. 
A

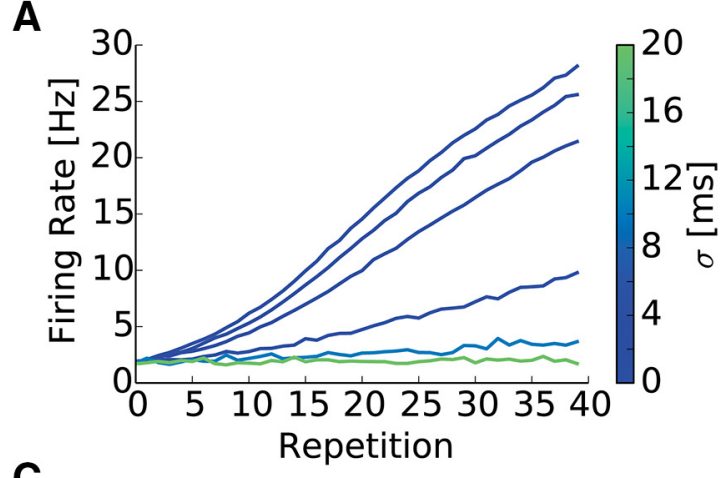

C

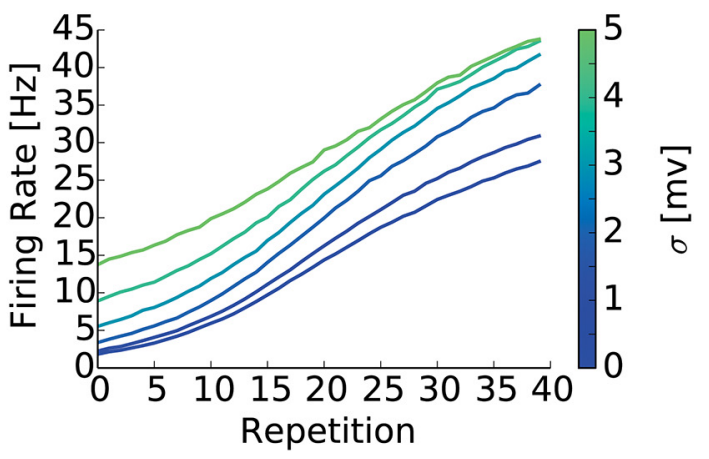

B

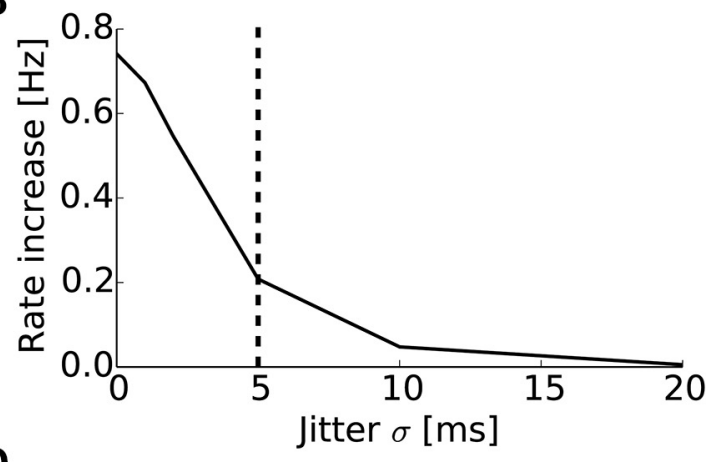

D

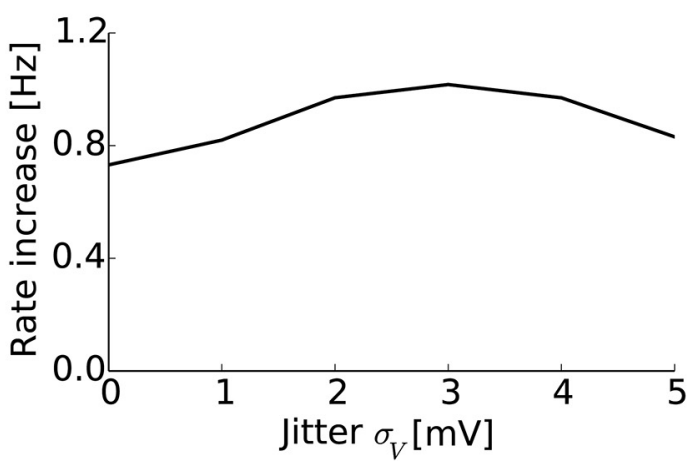

Figure 7. Fast learning relies on fine input correlations. $A$, Firing rate in response to the repeated pattern as a function of the number of repetitions, with random jitter introduced in spike timings $(\sigma 0,1,2,5,10$, and $20 \mathrm{~ms})$. $\boldsymbol{B}$, Rate increase per presentation (slope of the curves in $\boldsymbol{A})$ as a function of jitter SD. The membrane time constant is represented by the dashed line. $\boldsymbol{C}$, Firing rate in response to the repeated pattern as a function of the number of repetitions, when Gaussian noise of SD $\sigma_{\mathrm{V}}$ is added to the membrane potential dynamics. $\boldsymbol{D}$, Rate increase per presentation (slope of the curves in $C$ as a function of SD of the noise.

In addition, to provide a more complete picture of how noise can perturb the learning process, we added a Gaussian noise with a fixed voltage variance $\sigma_{\mathrm{V}}^{2}$ to the membrane potential dynamics of all neurons, representing intrinsic noise or external stimulusunrelated noise. As can be seen in Figure 7, $C$ and $D$, adding noise results in a global increase in the firing rate but has little effect on the rate increase with repeated pattern presentations.

Our analysis was done in a feedforward structure. Would the phenomenon still occur in a recurrently connected neural network? To answer this question, we considered a randomly connected network of 8000 excitatory neurons and 2000 inhibitory neurons, with connection probability $20 \%$ between any pair of neurons and conduction delays drawn uniformly between 0 and $5 \mathrm{~ms}$ (Fig. 8A). Excitatory neurons also receive external excitatory inputs from 8000 neurons, with $20 \%$ connection probability and random delays (see Materials and Methods). Feedforward external synapses are plastic as before, recurrent excitatory synapses within the network are also subject to classical STDP, and inhibitory to excitatory synapses within the network are subject to STDP according to a previously described mechanism (Vogels et al., 2011). We first run the model with random external inputs (same as in the feedforward model) until the network has reached equilibrium (500 s; Fig. 8B, left). Then, during a period of $100 \mathrm{~s}$, the same pattern is presented every $2 \mathrm{~s}$, as in the feedforward simulation (Fig. 8B, middle and right). After this period of $100 \mathrm{~s}$, we present random input to the network during $100 \mathrm{~s}$, then we present the pattern again (Fig. $8 \mathrm{~B}$, inset, average firing rate of the network over $2 \mathrm{~s}$ time bins during the whole simulation). The response of the network to the pattern increases rapidly during the first $100 \mathrm{~s}$ of presentation (Fig. $8 B$, middle), and this specific response is preserved after the $100 \mathrm{~s}$ pause, as in the feedforward situation (Fig. $8 B$, inset). Figure $8 C$ shows the response to the pattern (first half) and to the random input (second half) after learning. At equilibrium, neurons fire irregularly and receive substantial excitatory input from both external $\left(\left\langle\Sigma \mathrm{EPSP}_{\text {ext }}\right\rangle=10 \mathrm{mV}\right)$ and recurrent $\left(\left\langle\Sigma \mathrm{EPSP}_{\mathrm{rec}}\right\rangle=\right.$ $8.1 \mathrm{mV}$ ) connections (Fig. $8 D$ ). The balanced state is maintained by inhibitory plasticity (Vogels et al., 2011), such that inhibition and excitation approximately cancel each other. Figure $8 E$ shows the distribution of synaptic weights, with a bimodal distribution for excitatory weights, both from recurrent $w_{\text {ee }}$ and external $w_{\text {ext }}$ connections (inset; most synapses are weak). Note that, also as in the feedforward scenario, the reliability of the response to the pattern increases with the number of presentations (Fig. $8 F$ ). Thus, the phenomenon of fast learning is essentially conserved in a recurrently connected network, and we expect a stronger effect in networks with more synapses (here we used only $N_{\text {syn }}=1600$ external plastic synapses for computational reasons).

\section{Extension to other plasticity rules}

To illustrate the generality of our results, we also show in Figure 9 the results of simulations performed with two other plasticity rules, a pair-based plasticity rule with binary synapses and a triplet rule (Pfister and Gerstner, 2006). In experiments, changes in synaptic strength are reported for a large number of pairings, and we have implicitly assumed that each pre-post pairing yields a small change in synaptic strength. Alternatively, each pre-post pairing could yield a large change in synaptic strength, with low probability. This alternative interpretation makes little difference to our theoretical proposition, which relies on the effect on a large number of synapses (i.e., the average effect is what matters). This is 


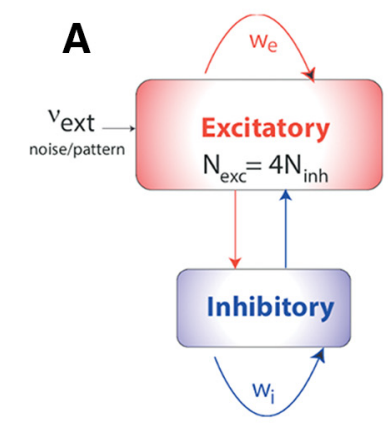

B

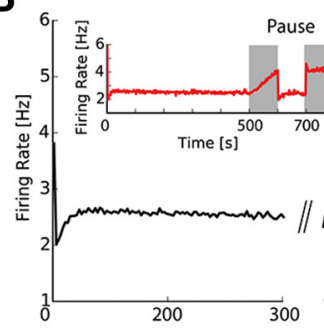

D

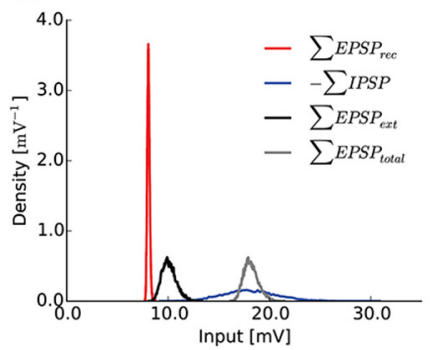

Pattern presentation
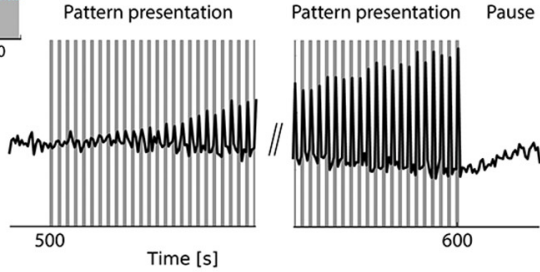

E

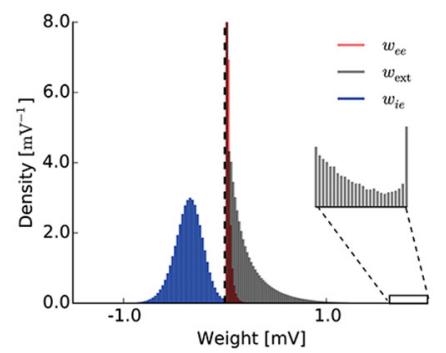

C

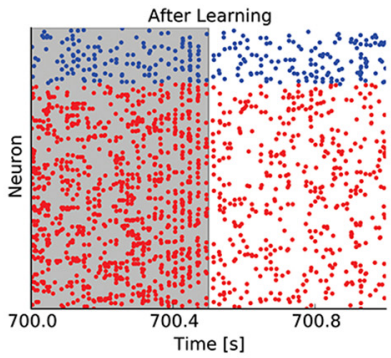

$\mathbf{F}$

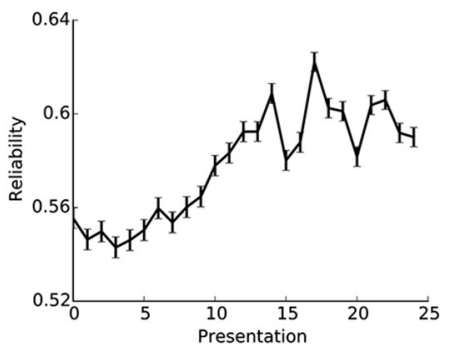

Figure 8. Learning in a balanced random network. $\boldsymbol{A}$, Structure of the random balanced network, with reciprocally coupled excitatory and inhibitory populations. $\boldsymbol{B}$, Evolution of the population firing rate as a function of time. The inset shows the global protocol of the simulation: after the network has reached an equilibrium (500 s), patterns are presented during $100 \mathrm{~s}$ chunks, once every $2 \mathrm{~s}$ (gray areas), with a pause in between. C, Spiking responses of the network over $1 \mathrm{~s}$ (blue, 100 inhibitory neurons; red, 400 excitatory neurons). The first half-second corresponds to the pattern. $D$, Distribution of the three types of inputs over all network neurons at equilibrium. $E$, Distribution of synaptic weights at equilibrium. The inset shows a zoom of the distribution near $w_{\max } . \boldsymbol{F}$, Evolution of the reliability of responses averaged over all neurons in the network, over the repeated presentations of the pattern.

demonstrated in Figure 9B, where we simulated a plasticity model with binary synapses (with either zero or maximal weight; see Materials and Methods), and pairings trigger low probability switches in the synapse state (average synaptic modifications were the same as in the original model). Specifically, plastic modifications are determined by the same presynaptic and postsynaptic traces $A_{\text {pre/post }}(t)$ and as in the previous model, but the traces determine the probability of state switching rather than the change in synaptic strength. As can be seen in Figure 9B, the same effect can be observed (although with more variability).

Figure $9 C$ shows the results of a simulation performed with a triplet rule adapted from Pfister and Gerstner (2006). This learning rule is able to capture more complex phenomena such as the frequency dependence of STDP (Sjöström et al., 2001). As shown in Figure 9, the results are qualitatively similar compared with those in Figure $2 A$. A few presentations of the pattern can trigger a significant change in the responses of the neurons. This is because the leverage effect observed here, whether with pair-based STDP or with the triplet learning rule, only depends on the fact that many synapses are potentiated by a single output spike ( of order $N$ ), whereas the magnitude of $V_{\mathrm{m}}$ fluctuations represents only a small number of PSPs (of order $\sqrt{N}$ ).

\section{Discussion}

This theoretical study has demonstrated that a fast increase in the response of a neuron to a new sensory stimulus, as observed in vivo (Yao et al., 2007), is consistent with the very small changes in synaptic strength observed in vitro (Markram et al., 1997; Bi and Poo, 1998; Wang et al., 2005). This apparent paradox is mainly caused by a leverage effect of order $\sqrt{N}$, where $N$ is the number of synapses, which occurs when the neuron is in a fluctuation-driven regime where excitation matches inhibition (also called balanced regime). This effect had not been seen in previous modeling studies (Masquelier et al., 2008; Masquelier et al., 2009; Klampfl and Maass, 2013), presumably because neurons were not initially in the fluctuation-driven regime, i.e., learning time actually reflected the time of convergence to that regime.

The main requirements for the effect are (1) a large number of synapses, (2) a fluctuation-driven regime, and (3) precise spike timing (within the neuron's temporal window of integration; Fig. 7). Pyramidal cells of the cortex and hippocampus receive, on average, $\sim 10,000$ synapses (Braitenberg and Schütz, 1991), giving a leverage effect of order 100. Evidence that cortical neurons operate in vivo in the fluctuation-driven regime comes from the irregularity of spike trains (Shadlen and Newsome, 1998; Nawrot et al., 2008) and from the characteristic shape of $V_{\mathrm{m}}$ distributions with a peak below threshold, reflecting a subthreshold mean current [Rossant et al. (2011), their Fig. 7] and measurements in barrel cortex (Crochet and Petersen, 2006), primary auditory cortex (DeWeese and Zador, 2006), primary visual cortex (Azouz and Gray, 1999), frontal cortex (Léger et al., 2005), and primary motor cortex (Brecht et al., 2004). There is additional evidence that inhibition is finely matched to excitation (Froemke et al., 2007; Okun and Lampl, 2008). Evidence for precise spike timing is more debated, but it should be noted that the requirement is not the reproducibility of absolute spike timing (relative to the stimulus) but of relative spike timing (or, more precisely, stimulus-specific synchrony). Many studies have reported such experimental evidence in response to natural stimuli presented in vivo (Petersen et al., 2001; Gollisch and Meister, 2008; Haider and McCormick, 2009; Bruno, 2011). Finally, there is evidence that neural activity is not stationary, with correlated firing in groups of neurons (Miller et al., 2014), contrary to what we considered in this study. However, those correlations were observed on a relatively long time scale ( $\sim 250 \mathrm{~ms}$ ), whereas only widespread correlations at the time scale of the integration time window 
(a few milliseconds) would have an impact on our assumptions (fluctuations of order $\sqrt{N}$ ).

An implicit assumption in our study is that single pre-post pairings should yield a change in synaptic strength. In experiments, changes in synaptic strength are reported for a large number of pairings, and here we have considered that the change is linear in the number of pairings. Linearity has been observed for 10-60 pairings (Froemke et al., 2006; Wittenberg and Wang, 2006), with saturation for larger numbers of pairings. Usually, observations of synaptic plasticity need several repetitions of a single pairing to observe an effect (Bi and Poo, 1998; Wang et al., 2005). This could be because the change for a single pairing is too small to be detected. Alternatively, it could be that there is a low probability of change in synaptic weight for a single pairing, rather than a small change in synaptic weight for each pairing. This would mean that on most trials with a single pairing, the synaptic weight would not change at all. As we have shown in Figure 9B, this alternative interpretation does not substantially alter our results. In vitro studies on synaptic plasticity also do not always report changes for low-frequency pairings (Sjöström et al., 2001). However, these negative results may be attributable to the neuron's voltage being at resting potential. In a depolarized state characteristic of cortical neurons in vivo, synaptic modifications are indeed seen with lowfrequency pairings (Cassenaer and Laurent, 2007; Harvey and Svoboda, 2007). Nonetheless, we cannot entirely rule out the possibility that a single pairing does not produce synaptic changes, in which case changes in neural responses after a single presentation would be attributable to some other mechanism than synaptic plasticity.

We have also shown that an additional leverage effect is provided by the presence of weak synapses that have small strength but can undergo the same plastic changes as active synapses. The proportion of weak synapses in cortical pyramidal cells or hippocampal neurons is thought to be substantial (Montgomery and Madison, 2002; Kerchner and Nicoll, 2008), and it has indeed been shown that the magnitude of LTP is similar for weak and strong synapses (Bi and Poo, 1998). In this study, we used a simple additive model of STDP (Bi and Poo, 1998; Song and Abbott, 2001). The actual physiological mechanism is likely to be more complex in neurons; for example, synaptic modifications may be influenced by voltage (Ngezahayo et al., 2000), pairing frequency (Sjöström et al., 2001), triplet effects (Wang et al., 2005), and initial synaptic strength (Bi and Poo, 1998). Several models may be able to capture those effects (Clopath et al., 2010; Yger and Harris, 2013), but even if this complexity would probably have a quantitative impact on the results, the main phenomenon does not depend on it (Fig. 9). The leverage effect observed here only depends on the fact that many synapses are potentiated by a single output spike (of order $N$ ), whereas the magnit- ude of $V_{\mathrm{m}}$ fluctuations represents only a small number of PSPs (of order $\sqrt{N}$ ). The same leverage effect supports the high sensitivity of neurons to coincident inputs (Rossant et al., 2011).

In a former neuronal network study with recurrent plasticity (Morrison et al., 2007), it was observed that synchronous stimulation resulted in de-balancing the network, which did not occur in this study for three reasons. First, we found no significant difference with and without plastic recurrent excitatory synapses in our study. Second and most importantly, delays were modeled as dendritic in the aforementioned study so that simultaneous spikes in two connected neurons result in a pre-post sequence at the synapse, triggering potentiation. In our simulations, delays were modeled as axonal, and the same configuration leads to depression. Third, inhibitory plasticity, which was included in our simulations, tends to restore the balance between excitation and inhibition (Vogels et al., 2011).

We also modeled synaptic inputs as currents rather than conductances, a simplifying assumption that makes analytical calculations simpler. The theory can be readily extended to the conductance case by replacing the balance equation with the equality of mean excitatory and inhibitory current at mean $V_{\mathrm{m}}$ (Richardson and Gerstner, 2005). As the excitatory reversal potential is high, including conductances should make little difference on the effect of excitatory inputs, but the effective membrane time constant of neurons would be lower. In our study, we implicitly included this effect by using a short membrane time constant $(5 \mathrm{~ms})$. We also assumed that the inputs were uncorrelated. The presence of stimulus-specific correlations be- 
tween excitatory inputs would be expected to increase the effect we have described. Indeed, in the fluctuation-driven regime, very small pairwise correlations (of order $1 / N$ ) result in a large increase in output firing rate, simply because there are many pairs of inputs (Rossant et al., 2011). Thus, we expect that stimulus-specific correlations would increase the learning rate. Nonstimulus-specific correlations, e.g., attributable to shared anatomical inputs, might be canceled by excitatory-inhibitory correlations (Renart et al., 2010).

Finally, our study addresses the retention of learned stimuli on the time scale of minutes or tens of minutes, but not on the time scale of days or weeks. Understanding how memory can be kept stable in the presence of spontaneous activity is still an open question. It has been shown that in networks with standard STDP, such memories cannot last (Morrison et al., 2007; Vogels et al., 2011; Litwin-Kumar and Doiron, 2014), except if some additional mechanisms are added such as nonlinearities (El Boustani et al., 2012) or bistability (Higgins et al., 2014). Therefore, longer time scales are thought to involve additional mechanisms such as synaptic tagging (Redondo and Morris, 2011), reflected in the late phase of LTP (Frey and Morris, 1997), which consolidate the initial changes, or neuromodulation (Matsuda et al., 2006). Moreover, the phenomenon discussed in this study may also underlie the reverberation of evoked activity patterns in spontaneous activity as observed in vivo (Kenet et al., 2003; Ikegaya et al., 2004; Yao et al., 2007; Han et al., 2008; Bermudez Contreras et al., 2013), which is thought to play a role in the consolidation of memories during sleep (Peyrache et al., 2009).

\section{References}

Agus TR, Thorpe SJ, Pressnitzer D (2010) Rapid formation of robust auditory memories: insights from noise. Neuron 66:610-618. CrossRef Medline

Azouz R, Gray CM (1999) Cellular mechanisms contributing to response variability of cortical neurons in vivo. J Neurosci 19:2209-2223. Medline

Bermudez Contreras EJ, Schjetnan AG, Muhammad A, Bartho P, McNaughton BL, Kolb B, Gruber AJ, Luczak A (2013) Formation and reverberation of sequential neural activity patterns evoked by sensory stimulation are enhanced during cortical desynchronization. Neuron 79: 555-566. CrossRef Medline

Bi GQ, Poo MM (1998) Synaptic modifications in cultured hippocampal neurons: dependence on spike timing, synaptic strength, and postsynaptic cell type. J Neurosci 18:10464-10472. Medline

Braitenberg V, Schütz A (1991) Anatomy of the cortex. Berlin: Springer.

Brecht M, Schneider M, Sakmann B, Margrie TW (2004) Whisker movements evoked by stimulation of single pyramidal cells in rat motor cortex. Nature 427:704-710. CrossRef Medline

Brunel N (2000) Dynamics of sparsely connected networks of excitatory and inhibitory spiking neurons. J Comput Neurosci 8:183-208. CrossRef Medline

Bruno RM (2011) Synchrony in sensation. Curr Opin Neurobiol 21:701708. CrossRef Medline

Caporale N, Dan Y (2008) Spike timing-dependent plasticity: a Hebbian learning rule. Annu Rev Neurosci 31:25-46. CrossRef Medline

Carey S, Bartlett E (1978) Acquiring a single new word. Papers Rep Child Lang Dev 15:17-29.

Cassenaer S, Laurent G (2007) Hebbian STDP in mushroom bodies facilitates the synchronous flow of olfactory information in locusts. Nature 448:709-713. CrossRef Medline

Clopath C, Büsing L, Vasilaki E, Gerstner W (2010) Connectivity reflects coding: a model of voltage-based STDP with homeostasis. Nat Neurosci 13:344-352. CrossRef Medline

Crochet S, Petersen CC (2006) Correlating whisker behavior with membrane potential in barrel cortex of awake mice. Nat Neurosci 9:608-610. CrossRef Medline

Destexhe A, Paré D (1999) Impact of network activity on the integrative properties of neocortical pyramidal neurons in vivo. J Neurophysiol 81: 1531-1547. Medline
Destexhe A, Rudolph M, Paré D (2003) The high-conductance state of neocortical neurons in vivo. Nat Rev Neurosci 4:739-751. CrossRef Medline

DeWeese MR, Zador AM (2006) Non-Gaussian membrane potential dynamics imply sparse, synchronous activity in auditory cortex. J Neurosci 26:12206-12218. CrossRef Medline

El Boustani S, Destexhe A (2009) A master equation formalism for macroscopic modeling of asynchronous irregular activity states. Neural Comput 21:46-100.21(1):46-100. CrossRef Medline

El Boustani S, Yger P, Frégnac Y, Destexhe A (2012) Stable learning in stochastic network states. J Neurosci 32:194-214. CrossRef Medline

Fahle M, Edelman S, Poggio T (1995) Fast perceptual learning in hyperacuity. Vision Res 35:3003-3013. CrossRef Medline

Frey U, Morris RG (1997) Synaptic tagging and long-term potentiation. Nature 385:533-536. CrossRef Medline

Fritz J, Shamma S, Elhilali M, Klein D (2003) Rapid task-related plasticity of spectrotemporal receptive fields in primary auditory cortex. Nat Neurosci 6:1216-1223. CrossRef Medline

Froemke RC, Tsay IA, Raad M, Long JD, Dan Y (2006) Contribution of individual spikes in burst-induced long-term synaptic modification. J Neurophysiol 95:1620-1629. CrossRef Medline

Froemke RC, Merzenich MM, Schreiner CE (2007) A synaptic memory trace for cortical receptive field plasticity. Nature 450:425-429. CrossRef Medline

Fusi S, Drew PJ, Abbott LF (2005) Cascade models of synaptically stored memories. Neuron 45:599-611. CrossRef Medline

Gerstner W, Kempter R, van Hemmen JL, Wagner H, Hemmen JV (1996) A neuronal learning rule for sub-millisecond temporal coding. Nature 383: 76-81. CrossRef Medline

Gollisch T, Meister M (2008) Rapid neural coding in the retina with relative spike latencies. Science 319:1108-1111. CrossRef Medline

Goodman DF, Brette R (2009) The brain simulator. Front Neurosci 3:192197. CrossRef Medline

Griffith JS, Horn G (1966) An analysis of spontaneous impulse activity of units in the striate cortex of unrestrained cats. J Physiol 186:516-534. CrossRef Medline

Gütig R, Aharonov R, Rotter S, Sompolinsky H (2003) Learning input correlations through nonlinear temporally asymmetric Hebbian plasticity. J Neurosci 23:3697-3714. Medline

Haider B, McCormick DA (2009) Rapid neocortical dynamics: cellular and network mechanisms. Neuron 62:171-189. CrossRef Medline

Han F, Caporale N, Dan Y (2008) Reverberation of recent visual experience in spontaneous cortical waves. Neuron 60:321-327. CrossRef Medline

Harvey CD, Svoboda K (2007) Locally dynamic synaptic learning rules in pyramidal neuron dendrites. Nature 450:1195-1200. CrossRef Medline

Higgins D, Graupner M, Brunel N (2014) Memory maintenance in synapses with calcium-based plasticity in the presence of background activity. PLoS Comput Biol 10:e1003834. CrossRef Medline

Hussain Z, McGraw PV, Sekuler AB, Bennett PJ (2012) The rapid emergence of stimulus specific perceptual learning. Front Psychol 3:226. CrossRef Medline

Ikegaya Y, Aaron G, Cossart R, Aronov D, Lampl I, Ferster D, Yuste R (2004) Synfire chains and cortical songs: temporal modules of cortical activity. Science 304:559-564. CrossRef Medline

Isaac JT, Nicoll RA, Malenka RC (1995) Evidence for silent synapses: implications for the expression of LTP. Neuron 15:427-434. CrossRef Medline

Kenet T, Bibitchkov D, Tsodyks M, Grinvald A, Arieli A (2003) Spontaneously emerging cortical representations of visual attributes. Nature 425: 954-956. CrossRef Medline

Kerchner GA, Nicoll RA (2008) Silent synapses and the emergence of a postsynaptic mechanism for LTP. Nat Rev Neurosci 9:813-825. CrossRef Medline

Klampfl S, Maass W (2013) Emergence of dynamic memory traces in cortical microcircuit models through STDP. J Neurosci 33:11515-11529. CrossRef Medline

Koch KW, Fuster JM (1989) Unit activity in monkey parietal cortex related to haptic perception and temporary memory. Exp Brain Res 76:292-306. Medline

Koulakov AA, Hromádka T, Zador AM (2009) Correlated connectivity and the distribution of firing rates in the neocortex. J Neurosci 29:3685-3694. CrossRef Medline

Laughlin SB (2001) Energy as a constraint on the coding and processing of 
sensory information. Curr Opin Neurobiol 11:475-480. CrossRef Medline

Léger JF, Stern EA, Aertsen A, Heck D (2005) Synaptic integration in rat frontal cortex shaped by network activity. J Neurophysiol 93:281-293. Medline

Litwin-Kumar A, Doiron B (2014) Formation and maintenance of neuronal assemblies through synaptic plasticity. Nat Commun 5:5319. CrossRef Medline

Markram H, Lübke J, Frotscher M, Sakmann B (1997) Regulation of synaptic efficacy by coincidence of postsynaptic APs and EPSPs. Science 275: 213-215. CrossRef Medline

Masquelier T, Guyonneau R, Thorpe SJ (2008) Spike timing dependent plasticity finds the start of repeating patterns in continuous spike trains. PLoS One 3:e1377. CrossRef Medline

Masquelier T, Guyonneau R, Thorpe SJ (2009) Competitive STDP-based spike pattern learning. Neural Comput 21:1259-1276. CrossRef Medline

Matsuda Y, Marzo A, Otani S (2006) The presence of background dopamine signal converts long-term synaptic depression to potentiation in rat prefrontal cortex. J Neurosci 26:4803-4810. CrossRef Medline

Miller JEK, Ayzenshtat I, Carrillo-Reid L, Yuste R (2014) Visual stimuli recruit intrinsically generated cortical ensembles. Proc Natl Acad Sci U S A 111:E4053-E4061. CrossRef Medline

Montgomery JM, Madison DV (2002) State-dependent heterogeneity in synaptic depression between pyramidal cell pairs. Neuron 33:765-777. CrossRef Medline

Morrison A, Aertsen A, Diesmann M (2007) Spike-timing-dependent plasticity in balanced random networks. Neural Comput 19:1437-1467. CrossRef Medline

Nabavi S, Fox R, Proulx CD, Lin JY, Tsien RY, Malinow R (2014) Engineering a memory with LTD and LTP. Nature 511:348-352. CrossRef Medline

Nawrot MP, Boucsein C, Rodriguez Molina V, Riehle A, Aertsen A, Rotter S (2008) Measurement of variability dynamics in cortical spike trains. J Neurosci Methods 169:374-390. CrossRef Medline

Ngezahayo A, Schachner M, Artola A (2000) Synaptic activity modulates the induction of bidirectional synaptic changes in adult mouse hippocampus. J Neurosci 20:2451-2458. Medline

Okun M, Lampl I (2008) Instantaneous correlation of excitation and inhibition during ongoing and sensory-evoked activities. Nat Neurosci 11: 535-537. CrossRef Medline

Petersen RS, Panzeri S, Diamond ME (2001) Population coding of stimulus location in rat somatosensory cortex. Neuron 32:503-514. CrossRef Medline

Peyrache A, Khamassi M, Benchenane K, Wiener SI, Battaglia FP (2009) Replay of rule-learning related neural patterns in the prefrontal cortex during sleep. Nat Neurosci 12:919-926. CrossRef Medline

Pfister JP, Gerstner W (2006) Triplets of spikes in a model of spike timingdependent plasticity. J Neurosci 26:9673-9682. CrossRef Medline

Poggio T, Fahle M, Edelman S (1992) Fast perceptual learning in visual hyperacuity. Science 256:1018-1021 Medline

Redondo RL, Morris RG (2011) Making memories last: the synaptic tagging and capture hypothesis. Nat Rev Neurosci 12:17-30. Medline

Renart A, de la Rocha J, Bartho P, Hollender L, Parga N, Reyes A, Harris KD
(2010) The asynchronous state in cortical circuits. Science 327:587-590. CrossRef Medline

Richardson MJ, Gerstner W (2005) Synaptic shot noise and conductance fluctuations affect the membrane voltage with equal significance. Neural Comput 17:923-947. CrossRef Medline

Rossant C, Leijon S, Magnusson AK, Brette R (2011) Sensitivity of noisy neurons to coincident inputs. J Neurosci 31:17193-17206. CrossRef Medline

Rubin N, Nakayama K, Shapley R (1997) Abrupt learning and retinal size specificity in illusory-contour perception. Curr Biol 7:461-467. CrossRef Medline

Rumpel S, Hatt H, Gottmann K (1998) Silent synapses in the developing rat visual cortex: evidence for postsynaptic expression of synaptic plasticity. J Neurosci 18:8863-8874. Medline

Seitz AR (2010) Sensory learning: rapid extraction of meaning from noise. Curr Biol 20:R643-R644. CrossRef Medline

Shadlen MN, Newsome WT (1998) The variable discharge of cortical neurons: implications for connectivity, computation, and information coding. J Neurosci 18:3870-3896. Medline

Sjöström PJ, Turrigiano GG, Nelson SB (2001) Rate, timing, and cooperativity jointly determine cortical synaptic plasticity. Neuron 32:11491164. CrossRef Medline

Softky WR, Koch C (1993) The highly irregular firing of cortical cells is inconsistent with temporal integration of random EPSPs. J Neurosci 13: 334-350. Medline

Song S, Abbott LF (2001) Cortical development and remapping through spike timing-dependent plasticity. Neuron 32:339-350. CrossRef Medline

Tan AYY, Chen Y, Scholl B, Seidemann E, Priebe NJ (2014) Sensory stimulation shifts visual cortex from synchronous to asynchronous states. Nature 509:226-229. CrossRef Medline

van Rossum MC, Bi GQ, Turrigiano GG (2000) Stable Hebbian learning from spike timing-dependent plasticity. J Neurosci 20:8812-8821. Medline

Vogels TP, Sprekeler H, Zenke F, Clopath C, Gerstner W (2011) Inhibitory plasticity balances excitation and inhibition in sensory pathways and memory networks. Science 334:1569-1573. CrossRef Medline

van Vreeswijk C, Sompolinsky H (1996) Chaos in neuronal networks with balanced excitatory and inhibitory activity. Science 274:1724-1726. CrossRef Medline

Wang HX, Gerkin RC, Nauen DW, Bi GQ (2005) Coactivation and timingdependent integration of synaptic potentiation and depression. Nat Neurosci 8:187-193. CrossRef Medline

Whitlock JR, Heynen AJ, Shuler MG, Bear MF (2006) Learning induces long-term potentiation in the hippocampus. Science 313:1093-1097. CrossRef Medline

Wittenberg GM, Wang SS (2006) Malleability of spike-timing-dependent plasticity at the CA3-CA1 synapse. J Neurosci 26:6610-6617. CrossRef Medline

Yao H, Shi L, Han F, Gao H, Dan Y (2007) Rapid learning in cortical coding of visual scenes. Nat Neurosci 10:772-778. CrossRef Medline

Yger P, Harris KD (2013) The Convallis rule for unsupervised learning in cortical networks. PLoS Comput Biol 9:e1003272. CrossRef Medline 